EPJ Web of Conferences 88, 00024 (2015)

DOI: $10.1051 /$ epjconf/ 20158800024

(C) Owned by the authors, published by EDP Sciences - SIF, 2015

\title{
Clustering in alpha conjugate nuclei
}

\author{
K. Schmidt ${ }^{1}$, E. J. Kim ${ }^{1,2}$, S. Wuenschel ${ }^{1}$, M. Barbui ${ }^{1}$, \\ J. B. Natowitz ${ }^{1}$, K. Hagel ${ }^{1}$, R. WAdA ${ }^{3,1}$, C. Bottosso ${ }^{1}$, \\ G. Giuliani ${ }^{1}$, L. Qin ${ }^{1}$, Z. Kohley ${ }^{1}$, A. Bonasera ${ }^{1}$, Z. Chen $^{1}$,

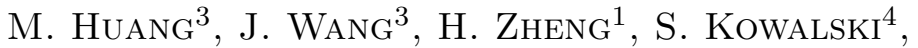 \\ M. R. D. Rodrigues ${ }^{1}$, D. Fabris ${ }^{5}$, S. Moretto ${ }^{5}$, S. Pesente ${ }^{5}$, \\ G. $\operatorname{Viesti~}^{5}$, M. Cinausero ${ }^{6}$, G. Prete ${ }^{6}$, G. Nebbia ${ }^{5}$, T. Keutgen ${ }^{7}$, \\ Y. El MASRI ${ }^{7}$, Z. MAJKA ${ }^{8}$ and Y. G. MA ${ }^{9}$ \\ ${ }^{1}$ Cyclotron Institute, Texas A\&M University, College Station, TX, USA \\ ${ }^{2}$ Chonbuk National University, Jeonju, Korea \\ ${ }^{3}$ IMP HIRFL, Chinese Academy of Sciences, Lanzhou, 730000, China \\ ${ }^{4}$ Institute of Physics, Silesia University, Katowice, Poland \\ ${ }^{5}$ Università di Padova and INFN, Sezione di Padova, Padova, Italy \\ ${ }^{6}$ INFN-Laboratori Nazionali di Legnaro, Legnaro (PD), Italy \\ ${ }^{7}$ Université Catholique de Louvain, B-1348 Louvain-Neuve, Belgium \\ ${ }^{8}$ Smoluchowski Inst. of Physics, Jagiellonian University, Kraków, Poland \\ ${ }^{9}$ Shanghai Institute of Nuclear Research, Shanghai 201800, China
}

\begin{abstract}
Characteristics of the decay of alpha like nuclei produced in various reactions with alpha conjugate nuclei are discussed. We observe a significant number of events in which a large fraction of the system breaks up into alpha-conjugate nuclei. Lighter fragments in the various decay channels appear to originate from a neck-like region between 4 and $7 \mathrm{~cm} / \mathrm{ns}$ whereas the heavier fragments appear to originate from velocities near the beam velocity. This behavior is consistent with a hierarchy effect that cannot be explained by statistical processes.
\end{abstract}

Alpha particles have long been proposed as possible building blocks of $\mathrm{N}=\mathrm{Z}$ nuclei [1]. It was recognized in this work that a cluster of $\alpha$-particles 


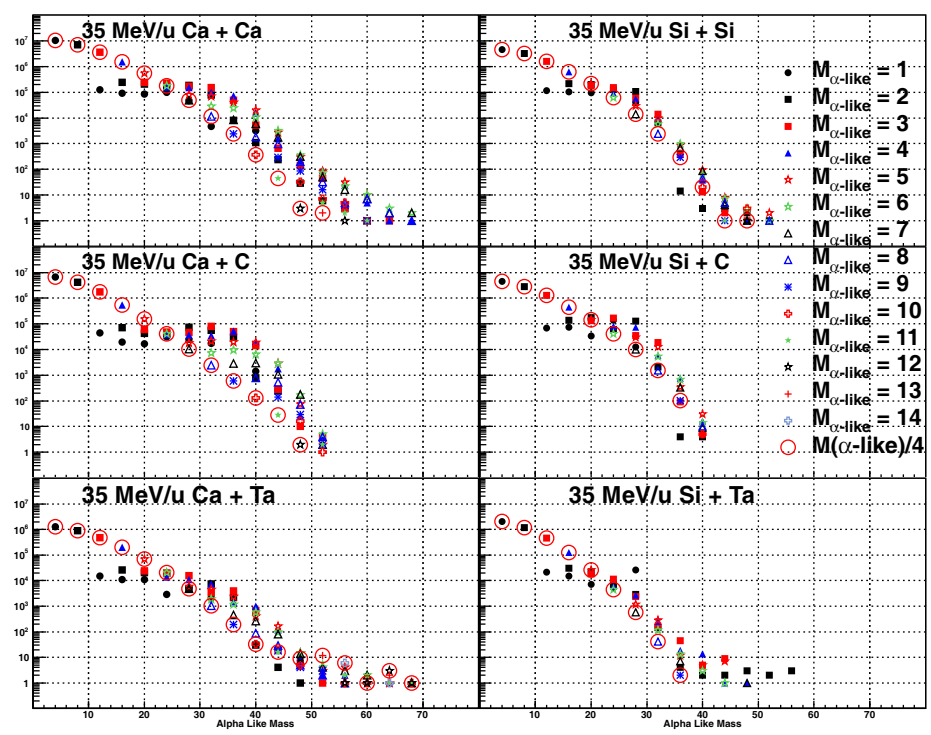

Figure 1: Probabilities of $\alpha$-like products as a function of $\alpha$-like mass.

could emerge as the excitation energy of the system was increased and different decay channels would depend on the degree of excitation of the system. That work explains observations in which $\alpha$ cluster states in light systems are observed at excitation energies near $\alpha$-decay thresholds [2,3]. A deviation of the decay of alpha cluster nuclei from statistical behavior is discussed in $[4,5]$.

We have pursued a study of the decay properties of $\alpha$-conjugate composite states by using a combination of beams and targets of $\alpha$-conjugate nuclei. In the following we show data from systems with a $35 \mathrm{MeV} / \mathrm{u} \mathrm{Ca}$ beam and a $35 \mathrm{MeV} / \mathrm{u}$ Si beam incident on different targets. The systems studied, in particular, are ${ }^{40} \mathrm{Ca}+{ }^{40} \mathrm{Ca},{ }^{40} \mathrm{Ca}+{ }^{12} \mathrm{C},{ }^{40} \mathrm{Ca}+{ }^{181} \mathrm{Ta},{ }^{28} \mathrm{Si}+$ ${ }^{28} \mathrm{Si},{ }^{28} \mathrm{Si}+{ }^{28} \mathrm{Si},{ }^{12} \mathrm{C}+{ }^{180} \mathrm{Ta}$. Note that each beam and each of the targets except Ta are alpha conjugate nuclei.

In the analysis the data were sorted as a function of the detected $\alpha$-like mass, that is, the sum of the masses of the detected products that are $\alpha$ conjugate nuclei. For a given $\alpha$-like mass, several different decay channels are possible [1]. In figure 1 we show a probablity distribution of the different decay channels as a function of $\alpha$-like mass. The different panels show the results for the systems under consideration. The different symbols indicate the multiplicity of alpha like products that were detected for the particular alpha like mass. The symbols with circles indicate the events where all of 


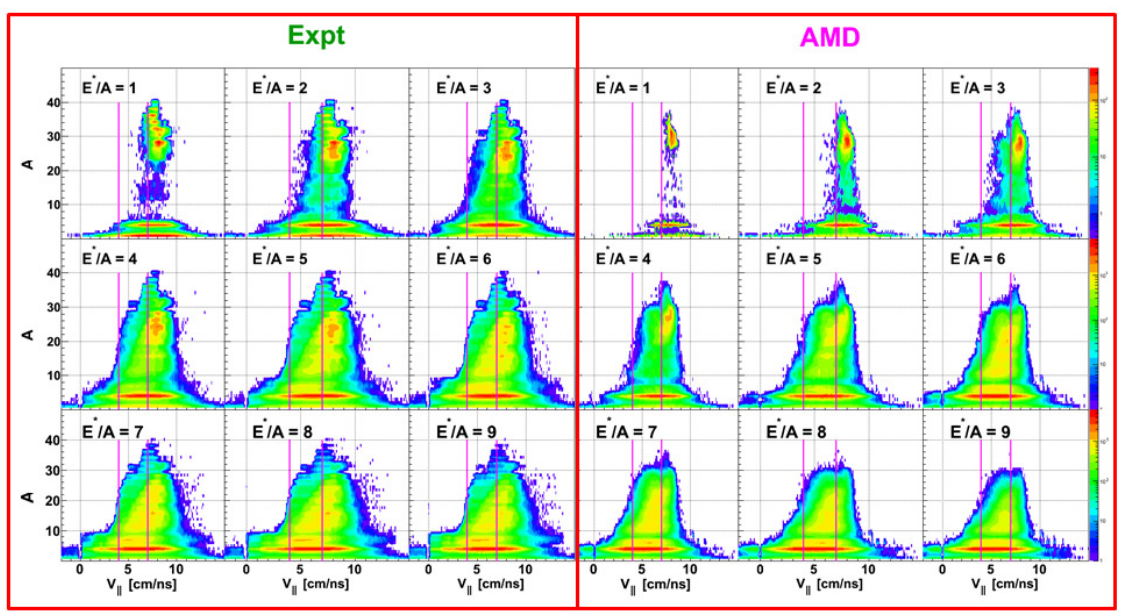

Figure 2: $\mathrm{A}$ vs $\mathrm{V}_{\|}$for $35 \mathrm{MeV} / \mathrm{u} \mathrm{Ca}+\mathrm{Ca}$. The vertical bars indiate the neck region between 4 and $7 \mathrm{~cm} / \mathrm{ns}$. Left: Experimental data. Right: AMD Calculations.

the alpha like mass is found in $\alpha$-particles.

We note in all of the systems studied that there is a significant number of events in which a large fraction of the event decays completely into products that are, themselves, $\alpha$-conjugate nuclei.

We next explore how these reactions evolve as a function of excitation energy. In figure 2 we present the distributions of mass vs parallel velocity of the detected products for different values of kinetic energy + Q-value (here called excitation energy). Figure 2 shows the results for $35 \mathrm{MeV} / \mathrm{u} \mathrm{Ca}+$ Ca. The left panel shows the results from the experimental data and the right hand side shows the results from an AMD calculation. We note for the low excitation energies that the majority of the products originate near the beam velocity of $8.1 \mathrm{~cm} / \mathrm{ns}$. As the excitation energy increases, the majority of the products evolve towards having an origin in the range of $4-7 \mathrm{~cm} / \mathrm{ns}$ which we label as the neck region. The AMD shows similar results.

To understand this neck region in more detail, we focus on events in which the detected alpha like mass is 40 in the $\mathrm{Ca}+\mathrm{Ca}$ system. Figure 3 (a) shows invariant velocity distributions for the different $\alpha$-like decay channels for these $\alpha$-like mass 40 events. The products of the different decay channels 

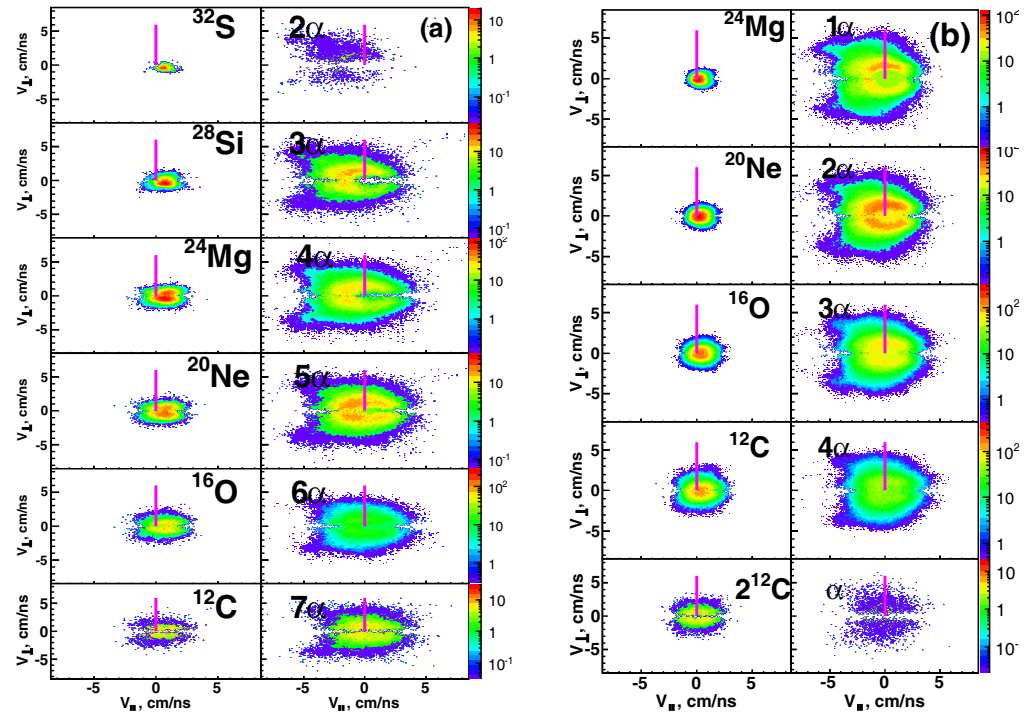

Figure 3: Invariant $\mathrm{V}_{\text {perp }}$ vs $\mathrm{V}_{\text {parallel }}$ for the various $\alpha$ decay channels. (a) 35 $\mathrm{MeV} / \mathrm{u} \mathrm{Ca}+\mathrm{Ca}$; (b) $35 \mathrm{MeV} / \mathrm{u} \mathrm{Si}+\mathrm{Si}$.

are transformed into the rest frame of the reconstructed alpha-like mass 40 nucleus. The vertical lines indicate the rest frame parallel velocity of the reconstructed emitting source. The left panel shows the heavy remnant and the right panel shows the alpha particles that make up the balance of the alpha-like mass 40 nucleus. The decay channels are indicated in the various panels. We note in this figure that the velocity spectra of the alpha particles, especially for the lower multiplicities, are peaked in the neck region at lower parallel velocities than the reconstructed decaying nucleus. On the other hand, the left panels show that the heavy partner travels at a velocity in excess of the reconstructed decaying nucleus. We also note that the trend becomes less prominent as the multiplicity of alpha particles increases and essentially vanishes for events with the highest multiplicities of 7 alpha particles.

Figure 3 (b) shows the same distributions for the $35 \mathrm{MeV} / \mathrm{u} \mathrm{Si}+\mathrm{Si}$ system. In these data we focus on events in which the alpha-like mass is 28 . We note the same trends as were discussed in the previous figure showing the $35 \mathrm{MeV} / \mathrm{u} \mathrm{Ca}+\mathrm{Ca}$ system which was gated on alpha-like mass 40 events. The trends are, perhaps, not as pronounced, but do tend to vanish as the 

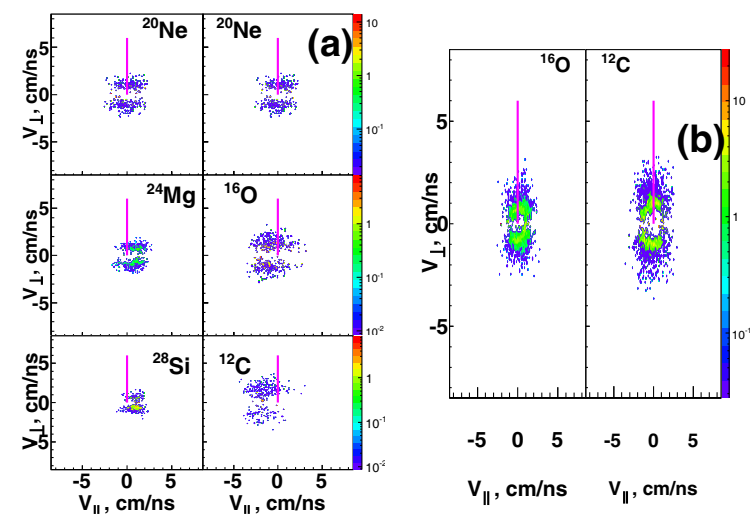

Figure 4: Invariant $\mathrm{V}_{\text {perp }}$ vs $\mathrm{V}_{\text {parallel }}$ for the various heavy-heavy $\alpha$-conjugate decay channels. (a) $35 \mathrm{MeV} / \mathrm{u} \mathrm{Ca}+\mathrm{Ca}$; (b) $35 \mathrm{MeV} / \mathrm{u} \mathrm{Si}+\mathrm{Si}$.

mulciplicity of alpha particles increases. This is probably due to the smaller size of the system where a lower multiplicity of the alpha particles is a larger fraction of the complete system.

In figure 4 (a) we show the other decay channels of the alpha-like mass 40 nucleus which consist of pairs of heavier alpha-like mass fragments. We observe a similar behavior in which the lighter fragment has the tendency to originate from the neck region being peaked at parallel velocities smaller than the parallel velocity of the decaying nucleus whereas the heavier fragments are found at velocities larger than the velocity of the decaying nucleus. This would suggest that the alpha particles in $n-\alpha$ events observed in the right panels of figure 3 (a) originate from the corresponding fragments on the right side of figure 4 (a).

Figure 4 (b) shows the similar distribution for the $35 \mathrm{MeV} / \mathrm{u} \mathrm{Si}+\mathrm{Si}$ system. Given the smaller size of the system, the number of decay combinations is limited to ${ }^{16} \mathrm{O}$ and ${ }^{12} \mathrm{C}$, but the trend, although smaller, is observed.

All of the systems studied show similar behavior. We take this to indicate the behavior of the alpha conjugate nuclei.

The decay patterns observed in figures 3 and 4 suggest a hierarchy of emission. Such a hierarchy has been observed in previous work [6]. To explore this we show distributions of observed mass vs parallel velocity. Figure 5 shows such distributions for the different decay channels for alphalike mass 40 events in the $35 \mathrm{MeV} / \mathrm{u} \mathrm{Ca}+\mathrm{Ca}$ system. We note in this figure that the heaviest fragment in the different decay channels always tends to 


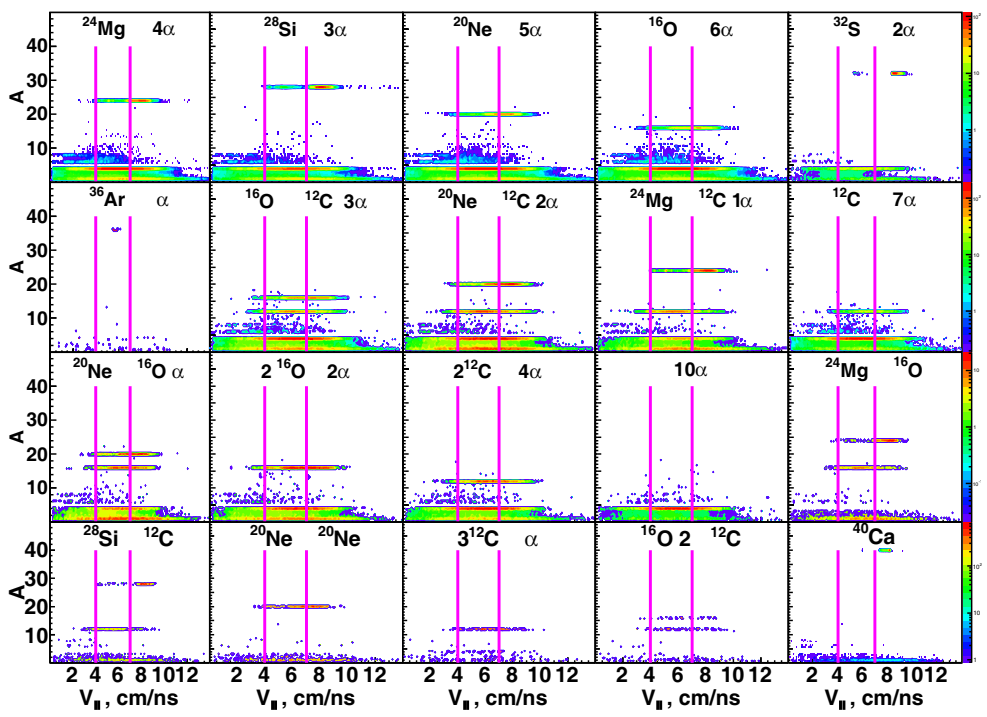

Figure 5: A vs $\mathrm{V}_{\text {parallel }} 35 \mathrm{MeV} / \mathrm{u} \mathrm{Ca}+\mathrm{Ca}$.

be observed at velocities larger than that of the neck region and that light particles tend to be observed as originating from the neck region. In the cases where there are two heavy fragments, the heaviest fragment is emitted at the higher velocity and the lighter one is emitted from the neck region. If we compare, for example, the ${ }^{24} \mathrm{Mg}_{-}{ }^{16} \mathrm{O}$ decay channel with the $2{ }^{16} \mathrm{O}$ $2 \alpha$ channel, we observe the ${ }^{16} \mathrm{O}$ at the light fragment in the first case to originate from the neck region whereas the ${ }^{16} \mathrm{O}$ in the second case being the heavy fragment (at least one of them) has more of a tendency to originate from velocities near the beam velocity and the $\alpha$-particles originate from the neck region. Similar trends are noted for all of the different decay channels.

Figure 6 shows the same distributions for the alpha-like mass 28 events in the $35 \mathrm{MeV} / \mathrm{u} \mathrm{Si}+\mathrm{Si}$ system. Although there is a smaller number of decay channels given the smaller size of the system, the results are similar. In particular, one can compare the ${ }^{16} \mathrm{O}-{ }^{12} \mathrm{C}$ channel with the $2{ }^{12} \mathrm{C}-\alpha$ channel. In the first the ${ }^{12} \mathrm{C}$ in predominantly located in the neck region and in the second the ${ }^{12} \mathrm{C}$ has a larger tendency to originate from higher velocities. There is significant part of the ${ }^{12} \mathrm{C}$ in the neck region, but there are two fragments and one of them probably originates from the neck region and the other from the higher velocity region. 


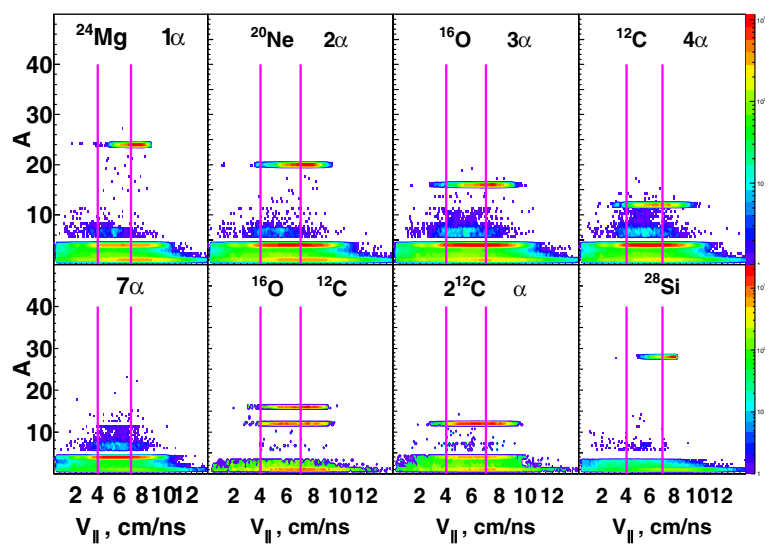

Figure 6: A vs $\mathrm{V}_{\text {parallel }} 35 \mathrm{MeV} / \mathrm{u} \mathrm{Si}+\mathrm{Si}$.

In summary, we have presented a study the decay patterns of alpha conjugate nuclei and have observed a significant number of events in which large fractions of the system breaks up into alpha-like products. The lighter products of the reactions appear to be emmitted from a neck like region between 4 and $7 \mathrm{~cm} / \mathrm{ns}$ where the heavier fragments predominantly originate from near the beam velocity. This is consistent with a dynamical hierarchy effect in which the lighter fragments trail the heavier fragments.

This work was supported by the United States Department of Energy under Grant \# DE-FG03- 93ER40773 and by The Robert A. Welch Foundation under Grant \# A0330.

\section{References}

[1] K. Ikeda, N. Tagikawa and H. Horiuchi, Prog. Theor. Phys. Suppl. E68, (1968) 464.

[2] M. Freer, Rep. on Prog. in Phys. 70 (2007) 2149.

[3] W. von Oertzen, M. Freer and Y. Kanada-En'yo, Phys. Rep. 432 (2007) 43.

[4] L. Morelli et al., hal-00975281 (2014). 
EPJ Web of Conferences

[5] Phys. Rev. C 87 (2013) 054614.

[6] J. Colin et al., Phys. Rev. C67 (2003) 064603. 